\title{
Effect of BN nanodots on the electronic properties of $a-$ and $\beta$-graphyne sheets: a density functional theory study
}

\author{
R. Majidi ${ }^{1}$ H. Eftekhari ${ }^{2} \cdot$ H. Bayat ${ }^{1} \cdot$ Kh. Rahmani $^{3} \cdot$ A. M. Khairogli ${ }^{4}$
}

Received: 13 July 2019 / Accepted: 21 September 2019 / Published online: 8 October 2019

(c) The Author(s) 2019

\begin{abstract}
The effect of BN nanodots with hexagonal shape on the electronic properties of $\alpha$ - and $\beta$-graphyne sheets is investigated. The structural and electronic properties of $\alpha$ - and $\beta$-graphyne sheets doped with BN nanodots are studied by using density functional theory. The cohesive energies of the systems indicate all considered structures are thermally stable. It is found that hexagonal BN nanodots can effectively open the band gap in $\alpha$ - and $\beta$-graphyne sheets. It means $\mathrm{BN}$ nanodots change $\alpha$ - and $\beta$-graphyne sheets from semimetal to semiconductor. The BN nanodots with different sizes are considered. It is found that band gaps of the studied $\alpha$ - and $\beta$-graphyne sheets doped with BN nanodots increase with the increase in the size of $\mathrm{BN}$ nanodots. Hence, $\alpha$ - and $\beta$-graphyne sheets doped with BN nanodots are promising materials for use in nanoelectronic devices based on semiconductors.
\end{abstract}

Keywords Density functional theory $\cdot$ Graphyne $\cdot$ BN nanodot $\cdot$ Band gap modification

\section{Introduction}

Two-dimensional (2D) carbon-based materials have attracted considerable attention in recent decades [1-5]. Examples of these 2D materials are graphene and graphyne sheets [1,2]. Graphene is a 2D hexagonal network of $s p^{2}$-bonded carbon atoms [3]. From the point of application, graphene is considered to be even more promising than other carbon-based nanostructures due to its extraordinary mechanical, thermal, electronic, and magnetic properties [3]. Like graphene, graphyne is a one-atom-thick layer of $\mathrm{C}$ atoms, but it contains both $s p$ - and $s p^{2}$-hybridized $\mathrm{C}$ atoms $[4,5]$. There are several types of graphyne. The most known graphyne are $\alpha-, \beta-, \gamma-$ and 6,6,12-graphyne sheets [5]. Interestingly, it is reported that $\alpha-, \beta-$, and 6,6,12-graphyne sheets posses amazing

H. Eftekhari

eftekhari.h@chmail.ir; h.eftekhari@srbiau.ac.ir

1 Department of Physics, Shahid Rajaee Teacher Training University, Lavizan, Tehran, Iran

2 Department of Physics, Science and Research Branch, Islamic Azad University, Tehran, Iran

3 Department of Chemistry, Kabul Polytechnic University, Kabul, Afghanistan

4 Department of Chemistry, Faryab University, Maymana, Faryab, Afghanistan electronic properties similar to graphene because of their peculiar band structure featuring so-called Dirac points and cones $[1,4,5]$. At a Dirac point, the valence and conduction bands cross each other at a single point at the Fermi level. It means that these sheets are semiconductors with zero band gap, or, alternatively, as metals with zero density of states (DOS) at the Fermi level [3-5].

Although graphene and graphyne are promising materials for use in nanoelectronic, the absence of a band gap limits their applications. Many strategies such as applying external electric field, producing nanoribbons, adsorbing molecules, chemical doping, and functionalization have been proposed to introduce a band gap in graphene and graphyne [6-23]. In particular, many studies have been focused on the modulation of the electronic properties of these sheets by doping [8-12]. $\mathrm{N}$ and $\mathrm{B}$ atoms are considered as the typical electron ( $n$-type) and hole ( $p$-type) doping elements since they are located in the periodic table one position behind and before from $\mathrm{C}$ atom, respectively. $\mathrm{B}$ - and $\mathrm{N}$-doped graphene and graphyne sheets are reported to be good semiconductors [8-12]. It is also found that BN co-doping is a process that opens a band gap in graphene and graphyne sheets, and changes the character of these sheets from a semimetal to a semiconductor [13-16]. Recently, graphene doped with $\mathrm{BN}$ nanodot has been realized experimentally $[16,18$, 19]. Effects of geometric shape and size of embedded BN 
nanodot on the electronic properties of $\mathrm{BN}$-doped graphene are studied theoretically $[16,18,19]$. It is found that the band gap of graphene increases with the size of the BN nanodot, regardless of the shape of $\mathrm{BN}$ nanodot. Motivated by these results, we have studied possibility of band gap opening in $\alpha$ - and $\beta$-graphyne sheets with BN nanodots doping based on density functional theory (DFT) in the present work.

\section{Computational details}

The calculations are carried out using DFT as implemented in OpenMX3.7 package [24]. The generalized gradient approximation (GGA) with Perdew-Burke-Ernzerhof (PBE) correction is employed for the exchange-correlation function [25]. The plane wave basis set is restricted by a cutoff energy of $100 \mathrm{Ry}$. All atomic structures are relaxed until the forces acting on each atom are smaller than $0.01 \mathrm{eV} / \mathrm{A}$. The k-point is set to be 41 along each high symmetry lines in the Brillouin zone. The charge transfer is calculated based on the Mulliken population analysis [26]. In order to have a better insight into the nature of bond characteristic, the charge density difference, $\rho_{\text {diff }}$, is calculated by [27]:

$\rho_{\text {diff }}=\rho_{\text {doped - sheet }}-\rho_{\text {pristine - sheet }}$

where $\rho_{\text {doped - sheet }}$ and $\rho_{\text {pristine - sheet }}$ denote the electron charge density of $\mathrm{BN}$-doped and pristine graphyne sheets, respectively.

Two types of graphyne, namely $\alpha$ - and $\beta$-graphyne, are studied. The $\alpha$-graphyne and $\beta$-graphyne sheets with $6 \times 6$ and $4 \times 4$ unit cells are considered (Fig. 1). The supercells of $\alpha$ - and $\beta$-graphyne sheets consist of $288 \mathrm{C}$ atoms. The periodic boundary conditions are applied to the supercell. The sheets are separated by $20 \AA$ to simulate an isolated graphyne sheet. It should be noted that we have calculated total energy to choose the size of the vacuum space. In Fig. 2, the total energy of $\alpha$ - and $\beta$-graphyne sheets as a function of the size of the vacuum space is shown. The minimum total energy is observed at the vacuum space of $20 \AA$. The BN nanodots with hexagonal shape and two different sizes are doped in the sheets. The sheets with BN nanodots are named $\mathrm{H}_{n}-(\mathrm{BN})_{m}$, where $n$ is the number of $\mathrm{BN}$ hexagonal rings and $m$ denotes the number of $\mathrm{BN}$ pairs doped in the graphyne sheets. For $\alpha$-graphyne, two $\mathrm{H}_{1}(\mathrm{BN})_{9}$ and $\mathrm{H}_{7}(\mathrm{BN})_{42}$ structures are studied (Fig. 3 ). In the case of $\beta$-graphyne, two $\mathrm{H}_{1}(\mathrm{BN})_{9}$ and $\mathrm{H}_{3}(\mathrm{BN})_{27}$ structures are considered (Fig. 4).

To discuss the stability of each structure, the cohesive energy is calculated. The cohesive energy per atom, $E_{\text {coh }}$, is defined as the difference between the energy of the structure and sum of the energies of the isolated atoms [28-31]. The cohesive energy of pristine graphyne sheets is defined as:

$E_{\text {coh }}=\frac{E_{\text {pristine - sheet }}-n_{\mathrm{C}} E_{C}}{n_{\mathrm{C}}}$
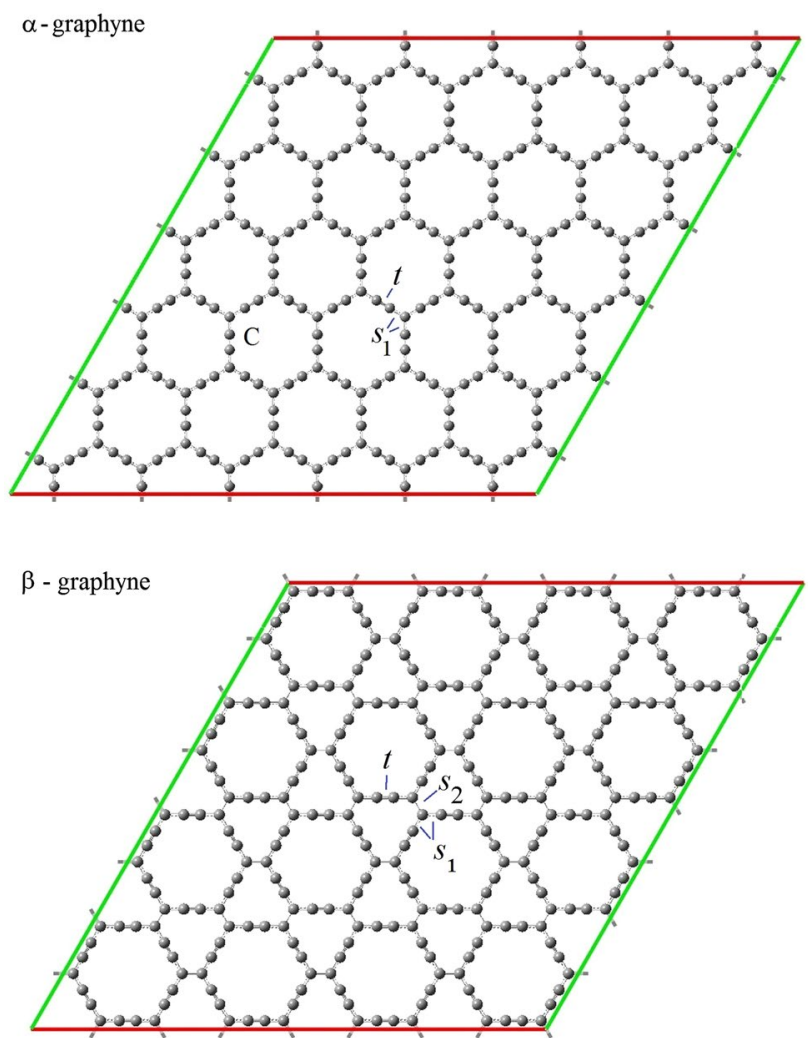

Fig. 1 Atomic structures of $\alpha$ - and $\beta$-graphyne sheets

For BN-doped graphyne sheets, it is given by:

$E_{\text {coh }}=\frac{E_{\text {doped - sheet }}-\left(n_{\mathrm{C}} E_{\mathrm{C}}+n_{\mathrm{N}} E_{\mathrm{N}}+n_{\mathrm{B}} E_{B}\right)}{n_{\mathrm{C}}+n_{\mathrm{N}}+n_{\mathrm{B}}}$

where $E_{\text {pristine - sheet }}$ and $E_{\text {doped - sheet }}$ are total energies of pristine and $\mathrm{BN}$-doped graphyne sheets, respectively. $E_{\mathrm{C}}, E_{\mathrm{N}}$, and $E_{\mathrm{B}}$ denote energies of $\mathrm{C}, \mathrm{N}$, and $\mathrm{B}$ atoms, respectively. $n_{\mathrm{C}}, n_{\mathrm{N}}$, and $n_{\mathrm{B}}$ are the number of $\mathrm{C}, \mathrm{N}$, and $\mathrm{B}$ atoms present in the sheets, respectively.

\section{Results and discussions}

The atomic structures of pristine $\alpha$ - and $\beta$-graphyne sheets are shown in Fig. 1. The graphyne sheets consist of both $s p$ - and $s p^{2}$-hybridized $\mathrm{C}$ atoms. The $\mathrm{C}-\mathrm{C}$ bond between two $s p$-hybridized $\mathrm{C}$ atoms is named $t$ (Fig. 1). The $\mathrm{C}-\mathrm{C}$ bond between $s p^{2}$ - and $s p$-hybridized $\mathrm{C}$ atoms is labeled by $s_{1}$, and the $\mathrm{C}-\mathrm{C}$ bond between two $s p^{2}$-hybridized $\mathrm{C}$ atoms is shown by $s_{2}$ (Fig. 1). In $\alpha$-graphyne, the single $\mathrm{C}-\mathrm{C}\left(s_{1}\right)$ and triple $\mathrm{C}-\mathrm{C}(t)$ bonds are 1.39 and $1.23 \AA$, respectively. For $\beta$-graphyne, the triple $\mathrm{C}-\mathrm{C}(t)$ bond is $1.23 \AA$, and the single $\mathrm{C}-\mathrm{C}\left(s_{1}\right.$ and $\left.s_{2}\right)$ bonds are 1.34 and $1.46 \AA$, respectively. These values are in good agreement with those reported in 

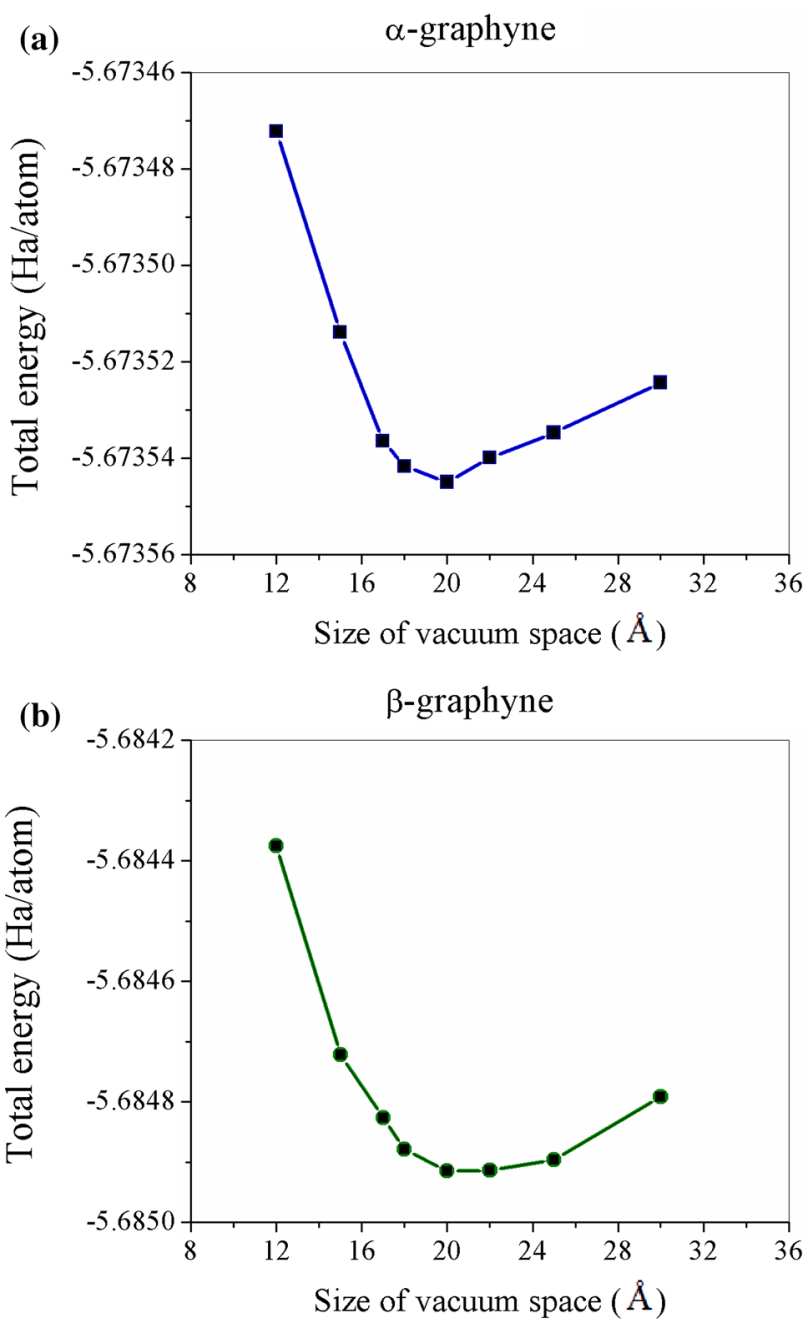

Fig. 2 Total energy of $\mathbf{a} \alpha$ - and $\mathbf{b} \beta$-graphyne sheets versus size of vacuum space

previous studies [5, 32]. Our calculated cohesive energies for $\alpha$ - and $\beta$-graphyne sheets are -4.87 and $-5.84 \mathrm{eV} /$ atom, respectively. Hence, $\beta$-graphyne with more negative cohesive energy is more stable than $\alpha$-graphyne. It is in good agreement with previous results that report the cohesive energy of graphyne decreases systematically as the ratio of $s p$-bonded carbon atoms increases [32].

The charge density difference diagram for pristine $\alpha$ - and $\beta$-graphyne sheets is illustrated in Fig. 5. Here, charge density isosurface value of $0.012 \mathrm{e} / \AA^{3}$ is chosen for the given diagrams. Electron loss and gain are represented by cyan and yellow color isosurfaces, respectively. It is observed that electron accumulation is mainly located within the $\mathrm{C}$ bonds, while electron depletion occurs around $\mathrm{C}$ atoms. It means that the $\mathrm{C}-\mathrm{C}$ bonds in pristine graphyne are covalent in nature.

To study the electronic properties of $\alpha$ - and $\beta$-graphyne sheets, their electronic band structures and DOS are
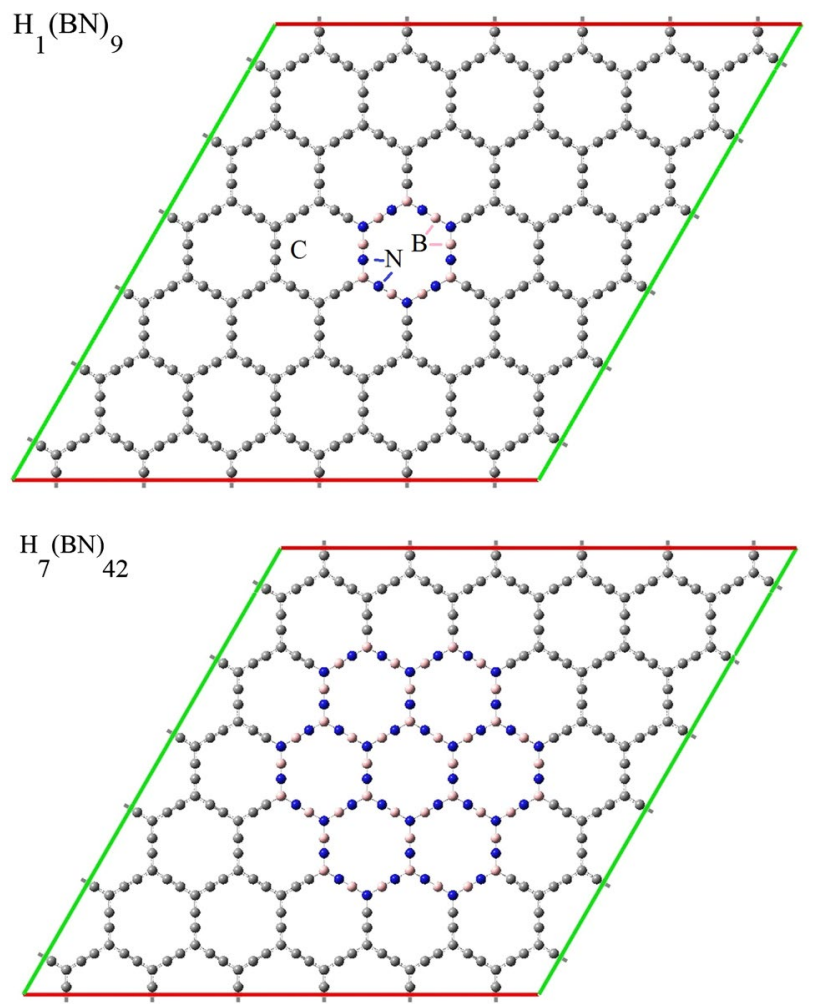

Fig. 3 Atomic structures of $\mathrm{H}_{1}-(\mathrm{BN})_{9}$ and $\mathrm{H}_{7}-(\mathrm{BN})_{42} \alpha$-graphyne sheets

calculated. As expected, the conduction and valence bands meet in a single point at the Fermi level (Fig. 6). The DOS is zero at the Fermi level. (The Fermi level is set at $0 \mathrm{eV}$.) These results mean $\alpha$ - and $\beta$-graphyne sheets have semimetallic properties as reported in the previous studies $[4$, $5,8,9]$.

The atomic structures of $\mathrm{H}_{1}-(\mathrm{BN})_{9}$ and $\mathrm{H}_{7}-(\mathrm{BN})_{42}$ $\alpha$-graphyne sheets are shown in Fig. 3. When BN nanodots are embedded in the $\alpha$-graphyne sheet, the $\mathrm{B}-\mathrm{C}$ and $\mathrm{N}-\mathrm{C}$ bonds are 1.51 and $1.34 \AA$, respectively. In both $\mathrm{H}_{1}-(\mathrm{BN})_{9}$ and $\mathrm{H}_{7^{-}}(\mathrm{BN})_{42} \alpha$-graphyne sheets, the $\mathrm{N}-\mathrm{C}$ bond lengths are shorter than the corresponding $\mathrm{C}-\mathrm{C}$ bond of the pristine $\alpha$-graphyne sheet $(1.39 \AA)$, while $\mathrm{B}-\mathrm{C}$ bond lengths are found to be longer. It is in close agreement with the previous reported results $[14,32]$. This is due to the fact that the higher electronegativity of $\mathrm{N}$ atoms attracts strongly the electron density compared with $\mathrm{B}$ atoms $[14,33]$. The $\mathrm{B}-\mathrm{N}(t)$ and $\mathrm{B}-\mathrm{N}\left(s_{1}\right)$ bonds are 1.27 and $1.40 \AA$, respectively. The cohesive energies of $\mathrm{H}_{1^{-}}(\mathrm{BN})_{9}$ and $\mathrm{H}_{7}-(\mathrm{BN})_{42}$ $\alpha$-graphyne sheets are calculated to understand the energetic stability of the sheets. The cohesive energies of $\mathrm{H}_{1}-(\mathrm{BN})_{9}$ and $\mathrm{H}_{7}-(\mathrm{BN})_{42} \alpha$-graphyne sheets are -4.95 and $-5.25 \mathrm{eV} /$ atom, respectively. The negative cohesive energies denote that $\mathrm{BN}$ doping in $\alpha$-graphyne sheet is an exothermic process, and $\mathrm{H}_{1}-(\mathrm{BN})_{9}$ and $\mathrm{H}_{7}-(\mathrm{BN})_{42} \alpha$-graphyne sheets can 

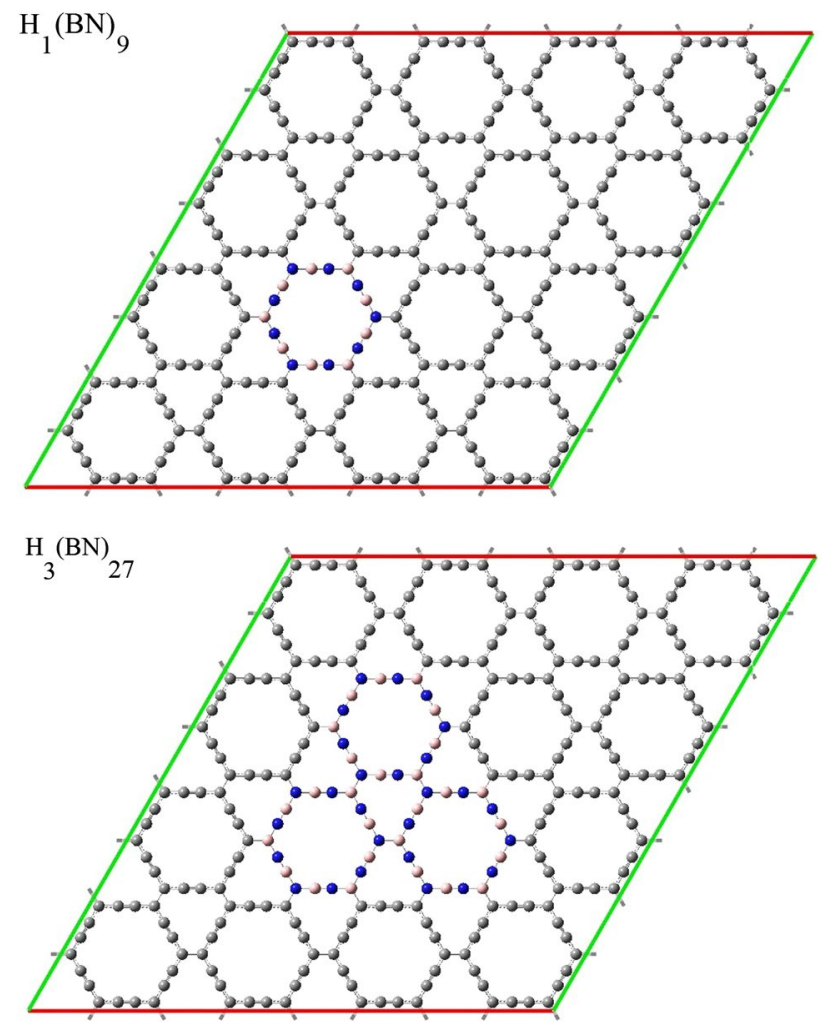

Fig. 4 Atomic structures of $\mathrm{H}_{1}-(\mathrm{BN})_{9}$ and $\mathrm{H}_{3}-(\mathrm{BN})_{27} \beta$-graphyne sheets

be produced experimentally. Here, $\mathrm{H}_{7}-(\mathrm{BN})_{42}$ with more negative cohesive energy is energetically more stable than $\mathrm{H}_{1}-(\mathrm{BN})_{9}$.

For $\beta$-graphyne sheet, two structures named $\mathrm{H}_{1}-(\mathrm{BN})_{9}$ and $\mathrm{H}_{3}-(\mathrm{BN})_{27}$ are studied (Fig. 4). In the presence of $\mathrm{BN}$ nanodots, the $\mathrm{B}-\mathrm{C}\left(s_{1}\right), \mathrm{N}-\mathrm{C}\left(s_{1}\right), \mathrm{B}-\mathrm{N}(t)$, and $\mathrm{B}-\mathrm{N}\left(s_{1}\right)$ bonds are $1.33,1.54,1.27$, and $1.40 \AA$, respectively. Similar to $\alpha$-graphyne, $\mathrm{B}-\mathrm{C}(\mathrm{N}-\mathrm{C})$ bond is longer (shorter) than the corresponding $\mathrm{C}-\mathrm{C}$ bonds. This is due to the difference between atomic radius of $\mathrm{B}, \mathrm{C}$, and $\mathrm{N}$ atoms which follows trend of $\mathrm{B}<\mathrm{C}<\mathrm{N}$. The cohesive energies of $\mathrm{H}_{1}-(\mathrm{BN})_{9}$ and $\mathrm{H}_{3}-(\mathrm{BN})_{27} \beta$-graphyne sheets are -5.26 and $-5.40 \mathrm{eV} /$ atom, respectively. The negative cohesive energies indicate that $\mathrm{BN}$ doping in $\beta$-graphene is an exothermic process and the considered structures are energetically stable. It is also found that $\mathrm{H}_{3}-(\mathrm{BN})_{27}$ is energetically more stable than $\mathrm{H}_{1}-(\mathrm{BN})_{9}$.

As an example, the charge density difference diagrams for $\mathrm{H}_{7}-(\mathrm{BN})_{42} \alpha$-graphyne and $\mathrm{H}_{3}-(\mathrm{BN})_{27} \beta$-graphyne sheets are shown in Fig. 7. A negative electron cloud around $\mathrm{N}$ atom resides as depicted by yellow isosurface, while electron deficiency takes place near B atom as depicted by cyan isosurface. The results of Mulliken charge analysis also support this finding. It is found that charge is transferred from $\mathrm{B}$ to $\mathrm{N}$ atom due to electronegativity difference of $\mathrm{B}$ and $\mathrm{N}$ atoms. It means $\mathrm{N}$ atoms gain and $\mathrm{B}$ atoms loss electrons.
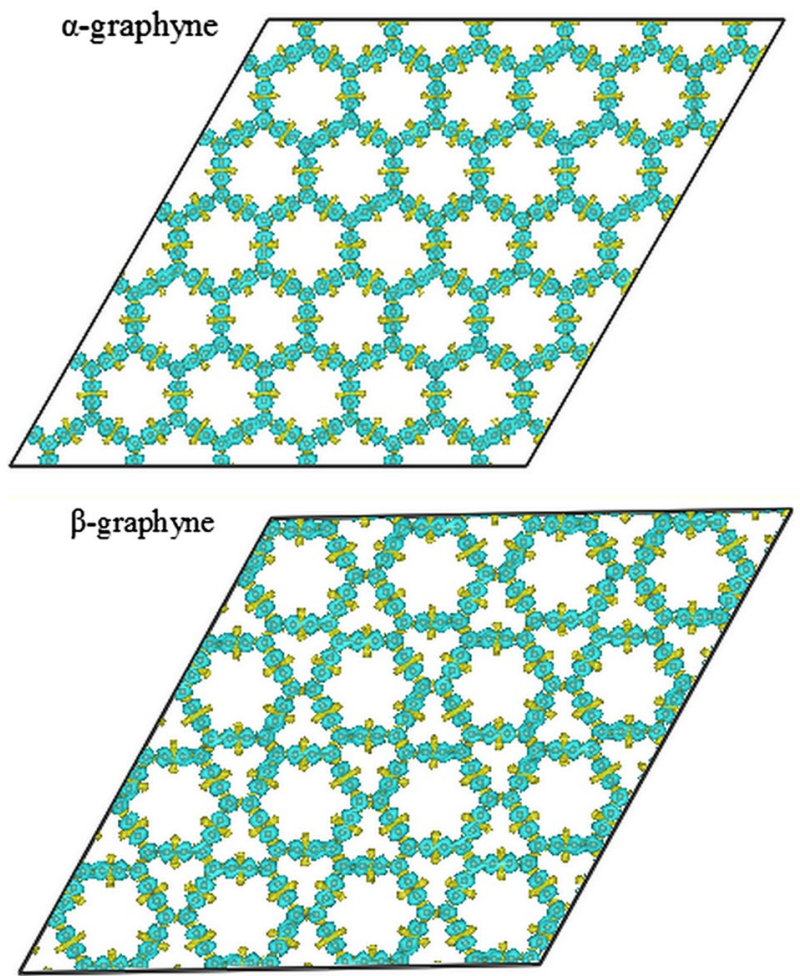

Fig. 5 Top view of charge density difference for pristine $\alpha$ - and $\beta$-graphyne sheets. (The yellow and cyan regions show the gain and loss of electron, respectively)

Thus, the $\mathrm{B}-\mathrm{N}$ bond has some ionic character, while $\mathrm{C}-\mathrm{C}$ bonds are covalent in $\mathrm{BN}$-doped graphyne sheets. The presence of $\mathrm{BN}$ is also modified slightly the charge distribution near the $\mathrm{C}$ atoms adjacent to $\mathrm{N}$ and $\mathrm{B}$ atoms. The Mulliken charge analysis shows 0.70 and $0.14 \mathrm{e}$ are transferred to $\mathrm{N}$ atom from $\mathrm{B}$ and adjacent $\mathrm{C}$ atoms; $0.06 \mathrm{e}$ is moved from $\mathrm{B}$ atom to adjacent $\mathrm{C}$ atom.

The electronic band structures and DOS of $\mathrm{H}_{1}-(\mathrm{BN})_{9}$ and $\mathrm{H}_{7}-(\mathrm{BN})_{42} \alpha$-graphyne sheets are also shown in Fig. 8. It is found that the electronic band structure of $\alpha$-graphyne is effectively modulated by $\mathrm{BN}$ doping. The results show that $\mathrm{H}_{1}-(\mathrm{BN})_{9}$ and $\mathrm{H}_{7}-(\mathrm{BN})_{42} \alpha$-graphyne sheets are semiconductors with direct band gaps of $0.12 \mathrm{eV}$ and $0.31 \mathrm{eV}$ at the $\Gamma$ point, respectively. It means that $\mathrm{BN}$ nanodots can effectively open a band gap in pristine $\alpha$-graphyne.

The electronic band structures and DOS of $\mathrm{H}_{1}-(\mathrm{BN})_{9}$ and $\mathrm{H}_{3}-(\mathrm{BN})_{27} \beta$-graphyne sheets are shown in Fig. 9. As seen, small band gaps of $0.02 \mathrm{eV}$ and $0.05 \mathrm{eV}$ are opened in $\mathrm{H}_{1}-(\mathrm{BN})_{9}$ and $\mathrm{H}_{3}-(\mathrm{BN})_{27} \beta$-graphyne sheets, respectively. The opened band gap and consequently the changes in the electronic properties of $\beta$-graphyne sheet in the presence of $\mathrm{BN}$ nanodots are not considerable.

The atomic structures of $\alpha$-graphyne- and $\beta$-graphyne-like $\mathrm{BN}$ sheets are illustrated in Fig. 10. In $\alpha$-graphyne-like BN sheet and in $\beta$-graphyne-like $\mathrm{BN}$ sheet, the $\mathrm{B}-\mathrm{N}$ bonds in the 


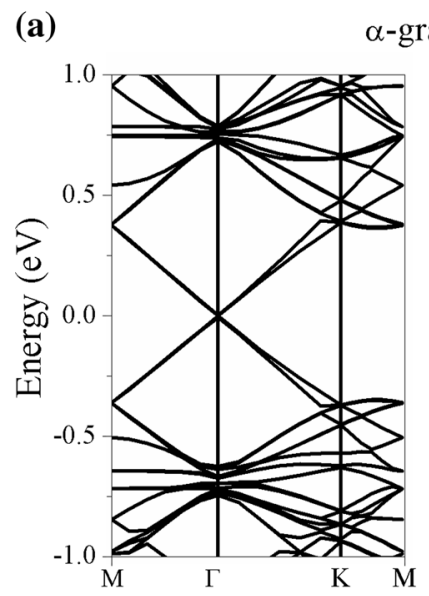

$\alpha$-graphyne

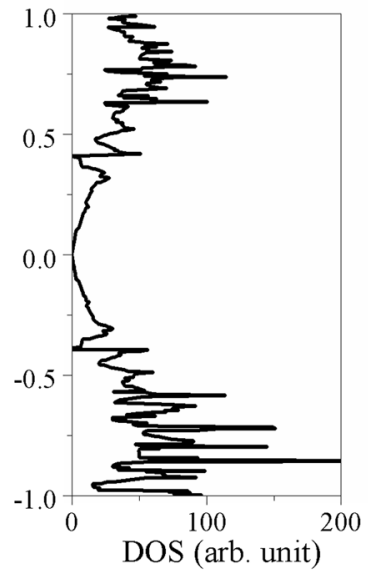

(b)

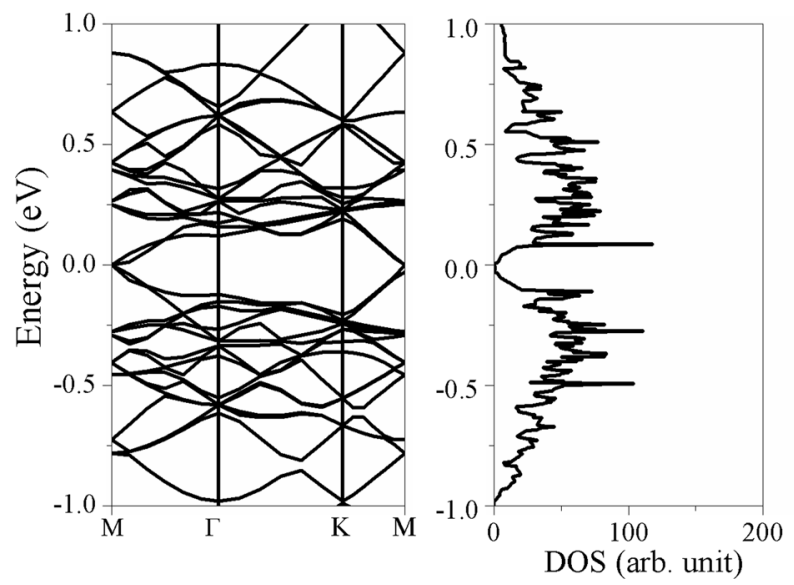

Fig. 6 Electronic band structures and DOS of $\mathbf{a} \alpha$ - and $\mathbf{b} \beta$-graphyne sheets

hexagons $\left(s_{2}\right)$ and at the middle of the chain $(t)$ are found to be 1.41 and $1.27 \AA$, respectively. The B-N $\left(s_{1}\right)$ bond with $\mathrm{B}$ atom at hexagon and $\mathrm{N}$ atom at chain is $1.39 \AA$, while the $\mathrm{B}-\mathrm{N}\left(s_{1}\right)$ bond between $\mathrm{N}$ atom at hexagon and $\mathrm{B}$ atom at linear chain is $1.40 \AA$. This difference is due to the different neighboring environments of $\mathrm{B}$ and $\mathrm{N}$ atoms [32]. The cohesive energies of these $\mathrm{BN}$ graphyne sheets are -6.23 and $-6.45 \mathrm{eV} /$ atom. Hence, $\beta$-graphyne-like $\mathrm{BN}$ sheet is more favorable than $\alpha$-graphyne-like $\mathrm{BN}$ sheet.

In Fig. 11, charge density difference diagrams for $\alpha$-graphyne- and $\beta$-graphyne-like BN sheets are shown. Here, $\mathrm{B}$ atoms loss electron and $\mathrm{N}$ atoms gain electron. The Mulliken charge analysis shows there are 0.71 e per $\mathrm{BN}$ pair transferring from $\mathrm{B}$ to $\mathrm{N}$ atoms. This is in good agreement with previous studies and confirms that the interaction between $\mathrm{B}$ and $\mathrm{N}$ atoms is ionic [36].

Calculations of the electronic band structures and DOS of $\alpha$-graphyne- and $\beta$-graphyne-like BN sheets (Fig. 12) show that these sheets are insulators with energy wide band
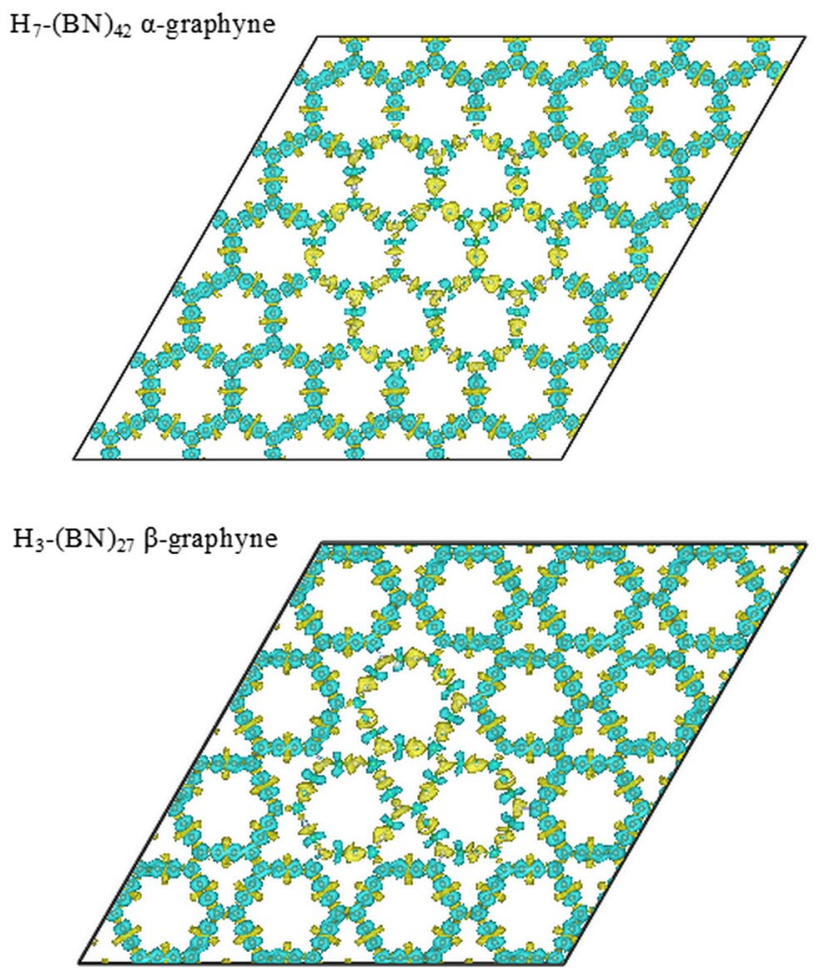

Fig. 7 Top view of charge density difference for $\mathrm{H}_{7}-(\mathrm{BN})_{42}$ $\alpha$-graphyne and $\mathrm{H}_{3}-(\mathrm{BN})_{27} \beta$-graphyne sheets. (The yellow and cyan regions show the gain and loss of electron, respectively)

gaps of 3.81 and $3.78 \mathrm{eV}$, respectively. The band gap of BN systems is attributed to the strong iconicity of $\mathrm{B}-\mathrm{N}$ bonds [34-36].

\section{Conclusions}

It has been previously showed that $\alpha$ - and $\beta$-graphyne sheets have semimetallic properties. Here, the electronic properties of $\alpha$ - and $\beta$-graphyne sheets doped with hexagonal BN nanodots are investigated by using density functional theory calculations. For comparison, the electronic properties of graphyne-like BN sheets are also studied. The thermal stability of these sheets was confirmed by calculation of the cohesive energy. The negative cohesive energies confirm that the considered structures are thermodynamically stable. The stability of these systems is in the order of $\mathrm{BN} \beta$-graphyne $>\mathrm{BN} \alpha$-graphyne $>\mathrm{H}_{3}$ $(\mathrm{BN})_{27} \beta$-graphyne $>\mathrm{H}_{1}-(\mathrm{BN})_{9} \beta$-graphyne $>\mathrm{H}_{7}-(\mathrm{BN})_{42}$ $\alpha$-graphyne $>\mathrm{H}_{1}-(\mathrm{BN})_{9} \alpha$-graphyne $>\beta$-graphyne $>\alpha$-gr aphyne. The results indicate that $\beta$-graphyne sheets are more stable than the $\alpha$-graphyne sheets. In addition, the stability increases with the increase in the size of BN nanodots. To find the effect of BN nanodots on the electronic 


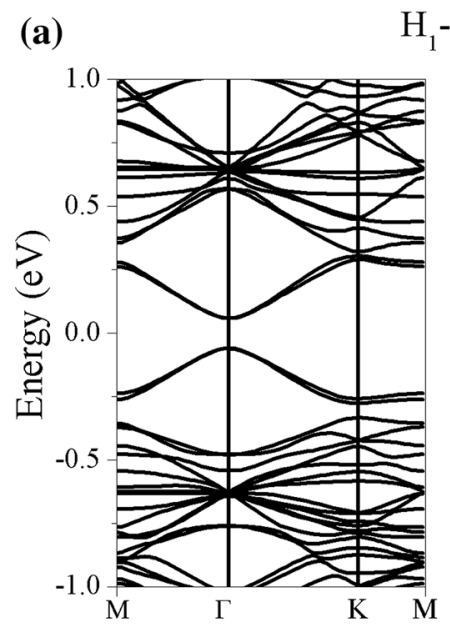

$$
\mathrm{H}_{1}-(\mathrm{BN})_{9}
$$

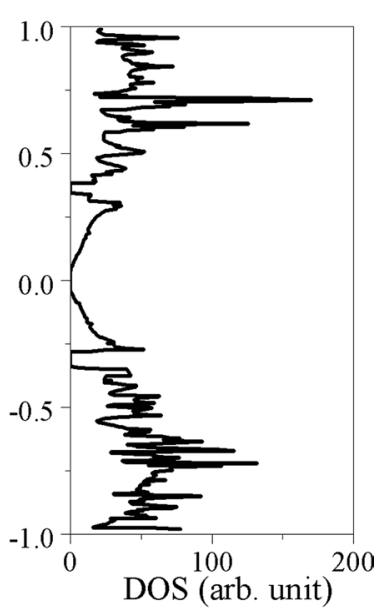

(b)

$\mathrm{H}_{7}-(\mathrm{BN})_{42}$
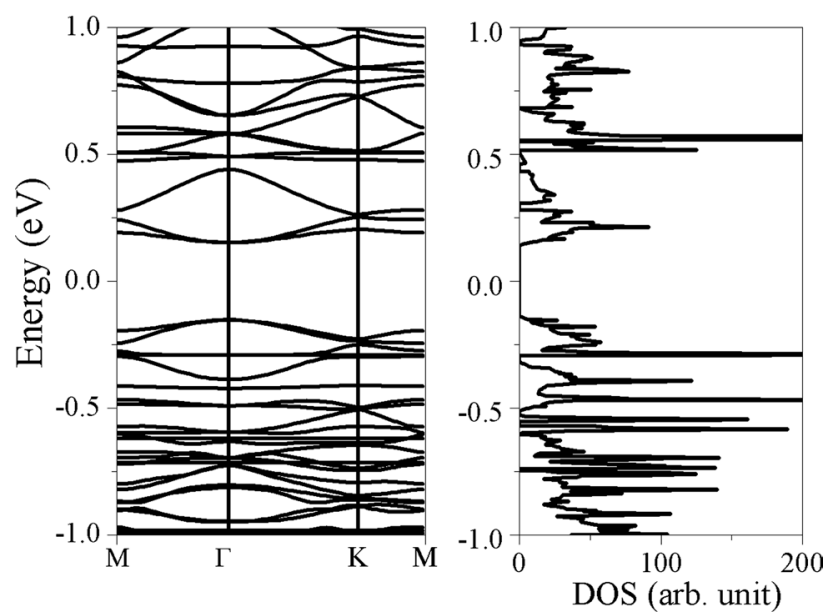

Fig. 8 Electronic band structures and DOS of a $\mathrm{H}_{1}-(\mathrm{BN})_{9}$ and $\mathbf{b}$ $\mathrm{H}_{7^{-}}(\mathrm{BN})_{42} \alpha$-graphyne sheets

properties of the sheets, the electronic band structure and DOS of the sheets are calculated. As expected, $\alpha$ - and $\beta$-graphyne sheets show semimetallic behavior, while $\alpha$ and $\beta$-graphyne-like $\mathrm{BN}$ sheets are insulator. The results reveal that $\mathrm{BN}$ nanodots could lead to a change in electron properties of $\alpha$ - and $\beta$-graphyne sheets. The BN nanodots open a band gap, and $\alpha$ - and $\beta$-graphyne sheets show semiconducting properties in the presence of the $\mathrm{BN}$ nanodots.

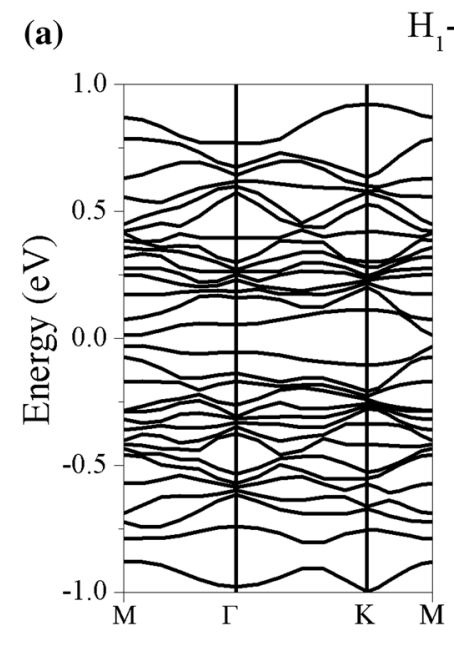

$\mathrm{H}_{1}-(\mathrm{BN})_{9}$
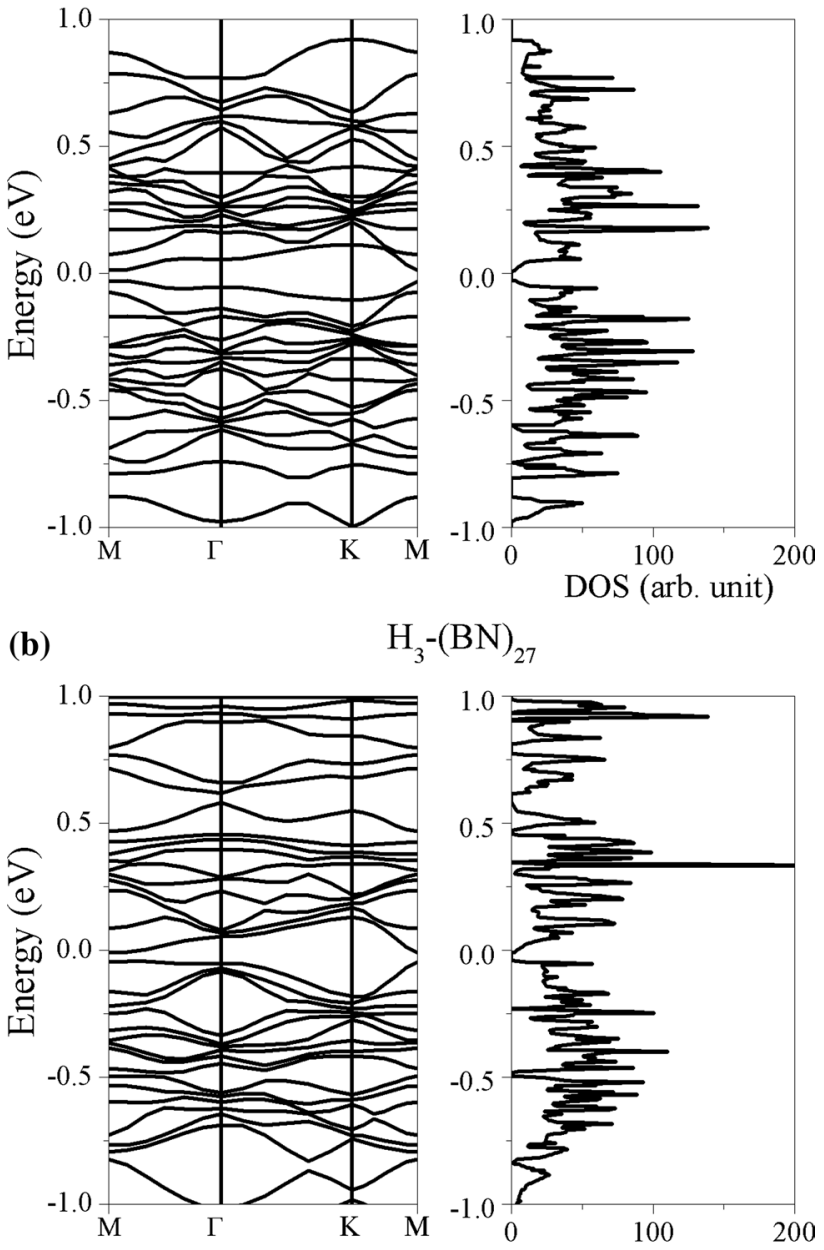
$-(\mathrm{BN})_{27}$

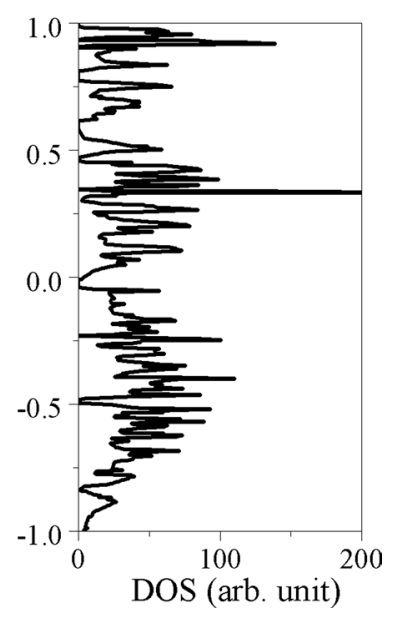

Fig. 9 Electronic band structures and DOS of $\mathbf{a} \mathrm{H}_{1}-(\mathrm{BN})_{9}$ and $\mathbf{b}$ $\mathrm{H}_{3}-(\mathrm{BN})_{27} \beta$-graphyne sheets

In the studied sheets, increasing the size of the BN nanodots increases the energy band gaps. Hence, BN doping in $\alpha$ - and $\beta$-graphyne sheets could be utilized to open and control the band gap in graphyne sheets. Our results suggest that $\alpha$ - and $\beta$-graphyne sheets with $\mathrm{BN}$ nanodots are more proper than pristine $\alpha$ - and $\beta$-graphyne sheets and $\alpha$ - and $\beta$-graphyne-like $\mathrm{BN}$ sheets for use in nanoelectronic devices due to their semiconducting properties. 

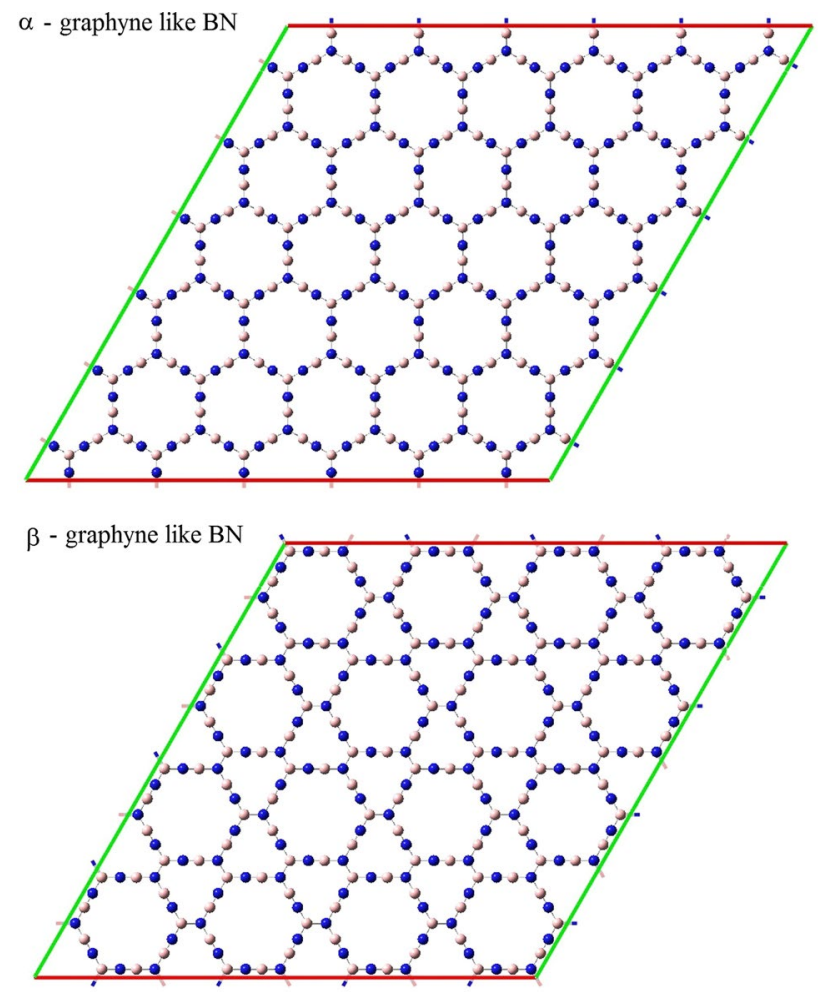

Fig. 10 Atomic structures of $\alpha$ - and $\beta$-graphyne-like BN sheets

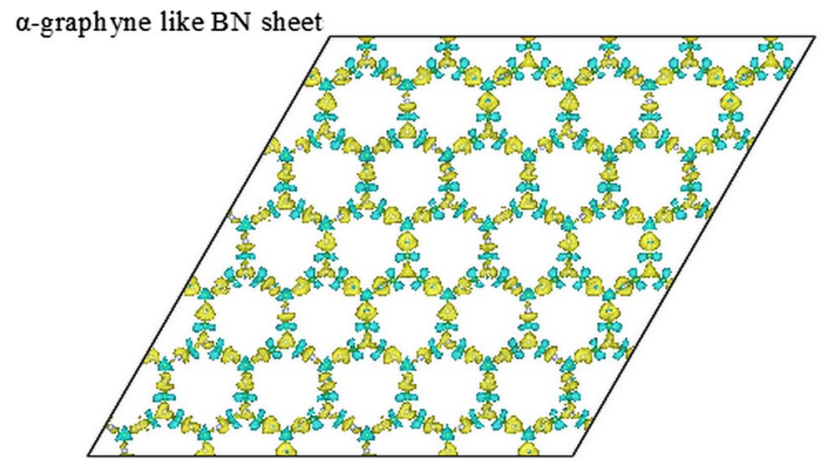

$\beta$-graphyne like BN sheet

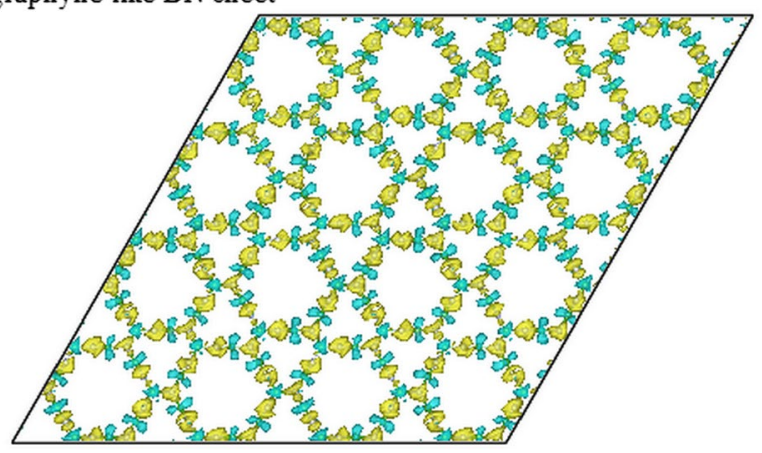

Fig. 11 Top view of charge density difference for $\alpha$ - and $\beta$-graphynelike BN sheets. (The yellow and cyan regions show the gain and loss of electron, respectively)

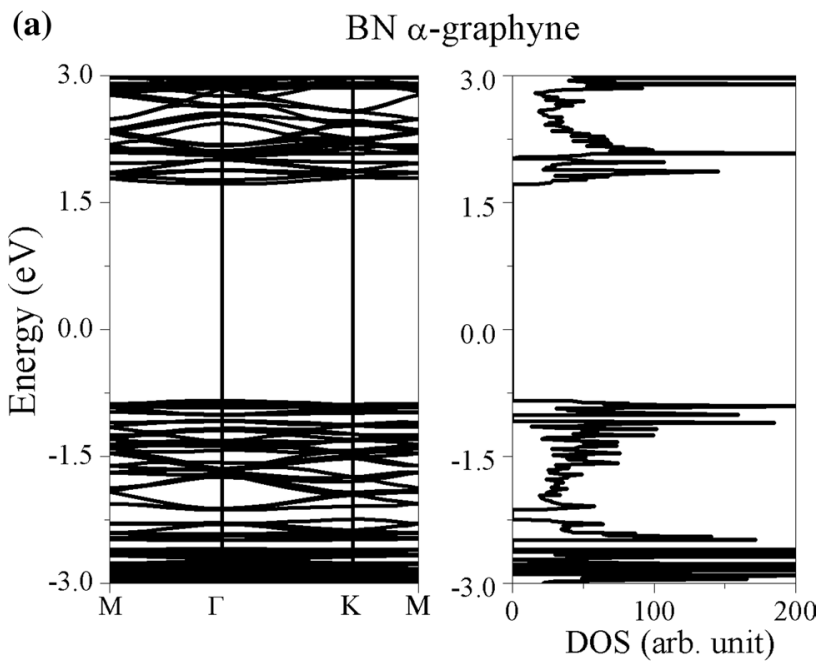

(b)

BN $\beta$-graphyne
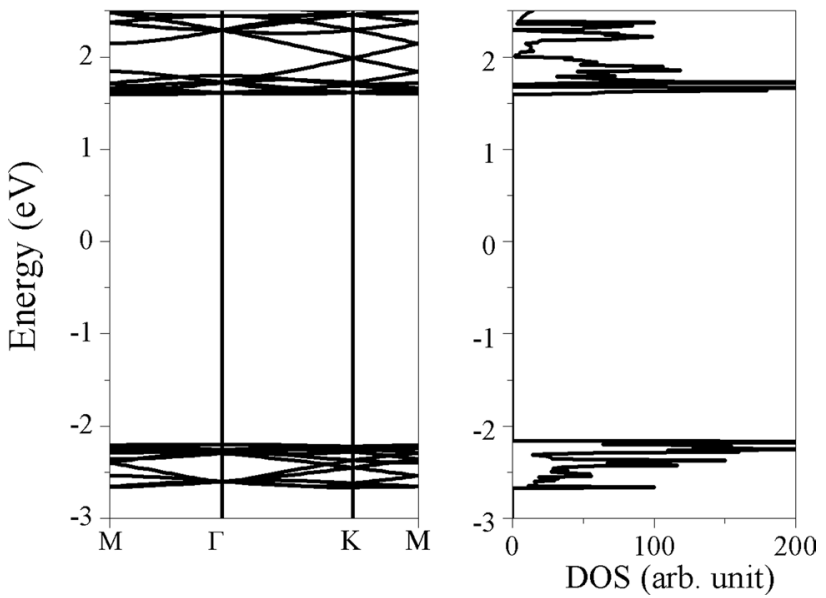

Fig. 12 Electronic band structures and DOS of $\mathbf{a} \alpha-$ and $\mathbf{b}$ $\beta$-graphyne-like $\mathrm{BN}$ sheets

Open Access This article is distributed under the terms of the Creative Commons Attribution 4.0 International License (http://creativeco mmons.org/licenses/by/4.0/), which permits unrestricted use, distribution, and reproduction in any medium, provided you give appropriate credit to the original author(s) and the source, provide a link to the Creative Commons license, and indicate if changes were made.

\section{References}

1. Geim, A.K., Novoselov, K.S.: The rise of graphene. Nat. Mater. 6, 183 (2007)

2. Balaban, A.T., Rentia, C.C., Ciupitu, E.: Chemical graphs. 6. Estimation of relative stability of several planar and tridimensional lattices for elementary carbon. Rev. Roum. Chim. 3, 231 (1968)

3. Tanaka, K., Iijima, S.: Carbon Nanotubes and Graphene, 2nd edn. Elsevier, Amsterdam (2014)

4. Kim, B.J., Choi, H.J.: Graphyne: hexagonal network of carbon with versatile Dirac cones. Phys. Rev. B 86, 115435 (2012) 
5. Malko, D., Neiss, C., Viñes, F., et al.: Competition for graphene: graphynes with direction-dependent Dirac cones. Phys. Rev. Lett. 108, 086804 (2012)

6. Zan, W.: Chemical functionalization of graphene by carbene cycloaddition: a density functional theory study. Appl. Surf. Sci. 311, 377 (2014)

7. Tan, J., He, X., Zhao, M.: First-principles study of hydrogenated graphyne and its family: stable configurations and electronic structures. Diam. Relat. Mater. 29, 42 (2012)

8. Majidi, R.: Effect of doping on the electronic properties of graphyne. NANO 6, 1350060 (2013)

9. Majidi, R., Karami, A.R.: Electronic properties of BN-doped bilayer graphene and graphyne in the presence of electric field. Mol. Phys. 111, 3194 (2013)

10. Majidi, R.: Electronic properties of T graphene-like C-BN sheets: a density functional theory study. Physica E 74, 371 (2015)

11. Rani, P., Jindal, V.K.: Designing band gap of graphene by B and N dopant atoms. RSC Adv. 3, 802 (2013)

12. Lazar, P., Zboril, R., Pumera, M., Otyepka, M.: Chemical nature of boron and nitrogen dopant atoms in graphene strongly influences its electronic properties. Phys. Chem. Chem. Phys. 16, 14231 (2014)

13. Kaloni, T.P., Joshi, R.P., Adhikari, N.P., Schwingenschlögl, U.: Band gap tuning in $\mathrm{BN}$-doped graphene systems with high carrier mobility. Appl. Phys. Lett. 104, 073116 (2014)

14. Yun, J., Zhang, Y., Xu, M., Yan, J., Zhao, W., Zhang, Z.: DFT study of the effect of BN pair doping on the electronic and optical properties of graphyne nanosheets. J. Mater. Sci. 52, 10294 (2017)

15. Rafique, M., Shuai, Y., Ahmed, I., Shaikh, R., Tunio, M.A.: Tailoring electronic and optical parameters of bilayer graphene through boron and nitrogen atom co-substitution; an ab initio study. Appl. Surf. Sci. 480, 463 (2019)

16. Ci, L., Song, L., Jin, C., Jariwala, D., Wu, D., Li, Y., Srivastava, A., Wang, Z.F., Storr, K., Balicas, L., Liu, F., Ajayan, P.M.: Atomic layers of hybridized boron nitride and graphene domains. Nat. Mater. 9, 430 (2010)

17. Ruiz-Puigdollers, A., Gamallo, P.: Synthesis of $\gamma$-graphyne by mechanochemistry and its electronic structure. Carbon 114, 301 (2017)

18. Enouz, S., Stéphan, O., Colliex, J.L., Loiseau, A.: C-BN patterned single-walled nanotubes synthesized by laser vaporization. Nano Lett. 7, 1856 (2007)

19. Xu, B., Lu, Y.H., Feng, Y.P., Lin, J.Y.: Density functional theory study of BN-doped graphene superlattice: role of geometrical shape and size. J. Appl. Phys. 108, 073711 (2010)

20. Tang, Q., Zhou, Z., Chen, Z.: Graphene-related nanomaterials: tuning properties by functionalization. Nanoscale 5, 4541 (2013)

21. Majidi, R.: Canadian, band gap modulation of graphyne via chemical functionalization: a density functional theory study. J. Chem. 94, 229 (2016)
22. Balog, R., Jorgensen, B., Nilsson, L., Andersen, M., Rienks, E., Bianchi, M., et al.: Bandgap opening in graphene induced by patterned hydrogen adsorption. Nat. Mater. 9, 315 (2010)

23. Huang, B.: Electronic properties of boron and nitrogen doped graphene nanoribbons and its application for graphene electronics. Phys. Lett. A 375, 845 (2011)

24. Ozaki, T., Kino, H., Yu, J., Han, M. J., Kobayashi, N., Ohfuti, M., Ishii, F., et al. User's Manual of OpenMX Version 3.7. http:// www.openmx-square.org

25. Perdew, J.P., Burke, K., Ernzerhof, M.: Generalized gradient approximation made simple. Phys. Rev. Lett. 77, 3865 (1996)

26. Mulliken, R.S.: Electronic population analysis on LCAO-MO molecular wave functions. I. J. Chem. Phys. 23, 1833 (1955)

27. Celaya, C.A., Muniz, J., Sansores, L.E.: Theoretical study of graphyne- $\gamma$ doped with $\mathrm{N}$ atoms: the quest for novel catalytic materials. Fuel 235, 384 (2019)

28. Singh, N.B., Bhattacharya, B., Sarkar, U.: A first principle study of pristine and BN-doped graphyne family. Struct. Chem. 25, 1695 (2014)

29. Ullah, S., Hussainb, A., Satoa, F.: Rectangular and hexagonal doping of graphene with B, N, and O: a DFT study. RSC Adv. 7, 16064 (2017)

30. Varghese, S.S., Swaminathan, S., Singh, K.K., Mittal, V.: Energetic stabilities, structural and electronic properties of monolayer graphene doped with boron and nitrogen atoms. Electronics 5, 91 (2016)

31. Bhattacharya, B., Sarkar, U., Singh, N.B.: The effect of boron and nitrogen doping in electronic, magnetic, and optical properties of graphyne. J. Phys. Chem. C 120, 26793 (2016)

32. Shin, H., Kang, S., Koo, J., Lee, H., Kim, J., Kwon, Y.: Cohesion energetics of carbon allotropes: Quantum Monte Carlo study. J. Chem. Phys. 40, 11470 (2014)

33. Puigdollers, A.R., Gamallo, P.: DFT study of the role of N- and B-doping on structural, elastic and electronic properties of $\alpha$-, $\beta$ - and $\gamma$-graphyne. Carbon 114, 301 (2017)

34. Topsakal, M., Akturk, E., Ciraci, S.: A comprehensive list of current references on layered and single-layer $\mathrm{BN}$ is given in this paper. Phys. Rev. B 79, 115442 (2009)

35. Park, C.H., Louie, S.G.: Energy gaps and stark effect in boron nitride nanoribbons. Nano Lett. 8, 2200 (2008)

36. Majidi, R., Rabczuk, T.: Structural and electronic properties of $\mathrm{BN}$ co-doped and BN analogue of twin graphene sheets: a density functional theory study. J. Phys. Chem. Solids 135, 109115 (2019)

Publisher's Note Springer Nature remains neutral with regard to jurisdictional claims in published maps and institutional affiliations. 